\title{
A Case of Post-partum Spontaneous Coronary Artery Dissection with Pulmonary Embolism: Survival with Thrombectomy and Stent Implant
}

\author{
Zin W Myint ${ }^{1}$, Kyaw Z Thein ${ }^{1}$, Khaing Moe ${ }^{2}$, Muhammad H Dogar ${ }^{2}$ and Robert Dobbin Chow ${ }^{3}$ \\ ${ }^{1}$ Medstar Good Samaritan Hospital Baltimore, USA \\ ${ }^{2}$ Kingsbrook Jewish Medical Center, USA \\ ${ }^{3}$ University of Maryland Medical Center Midtown Campus, USA
}

\begin{abstract}
Spontaneous Coronary Artery Dissection (SCAD) is a rare life-threatening cause of acute coronary syndrome. It can affect young patients without atherosclerotic risk factors, particularly women in the antepartum or early post-partum period, as well as geriatric patients at high risk for atherosclerotic disease. The pathogenesis linking SCAD with pregnancy has not been fully elucidated. The few reported cases of SCAD in the setting of concomitant Pulmonary Embolism (PE) may highlight a potential mechanism in the pregnant or postpartum woman. Heretofore, cases of SCAD with the setting of pulmonary embolism have all been treated conservatively with medical therapy. We herein report a case of SCAD in the left anterior descending artery resulting in Non-ST elevation myocardial infarction and low ejection fraction associated with an acute pulmonary embolism in a young post-partum patient who was treated successfully with thrombectomy followed by stent placement. Her cardiac function returned to normal within six months with no recurrence of symptoms. To our knowledge, this is the first case of SCAD in the setting of pulmonary embolism who was successfully treated with thrombectomy and stent placement.
\end{abstract}

Keywords: Spontaneous coronary artery dissection; NSTEMI; Pulmonary embolism; Thrombectomy and stent placement

\section{Introduction}

Spontaneous Coronary Artery Dissection (SCAD) is a rare lifethreatening cause of acute coronary syndrome and sudden cardiac death. It often affects younger, otherwise healthy patients [1-5], but can also be associated with atherosclerotic plaque rupture, chest trauma, or connective tissue diseases such as Marfan's syndrome and EhlersDanlos type IV. We herein report a case of SCAD associated with acute pulmonary embolism occurring in a postpartum patient who was successfully treated with thrombectomy and stent placement. This case focuses attention on a potential mechanism of disease for SCAD and introduces a novel therapeutic approach.

\section{Case Report}

A 35-year old G6P4A2 woman with a history of migraine headaches and sickle cell trait delivered a healthy infant through an uncomplicated normal spontaneous vaginal birth. Two weeks postpartum, she developed acute shortness of breath on exertion, followed by sharp left sub-sternal chest pain radiating to her back. The chest pain occurred twice, was not related to exertion, lasted 15 to 30 minutes per episode, and was associated with palpitations. She denied diaphoresis, nausea, vomiting, leg swelling, abdominal pain, trauma, or previous surgery. There was no history of prior similar episodes or family history of premature coronary heart disease. She had no history of hypertension, diabetes, or dyslipidemia, and there was no history of use of tobacco products, alcohol, or illicit drugs. She was not taking oral contraceptive pills.

On initial examination, the vital signs were $\mathrm{T} 99.8 \mathrm{~F}$, blood pressure $124 / 88 \mathrm{mmHg}$, pulse 95 beats per minute, and respiratory rate 16 breaths per minute. Her oxygen saturation on room air was $98 \%$ and she was not in acute distress. Cardiac examination revealed normal S1 and S2 heart sounds with no murmurs or gallops.

There were no rales, jugular venous distention, or accentuation of P2. The remainder of the physical examination was unremarkable with the exception of bilateral engorged tender breasts and a soft, lax non-tender abdomen with a palpable uterus consistent with 16 weeks gestational size.
Initial laboratory tests, including complete blood count with differential, basic metabolic profile, and urinalysis, were all within normal limits with the exception of a mild leukocytosis with WBC of

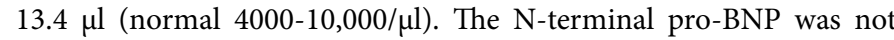
elevated and urine toxicology was negative. ANA was negative, antidouble stranded DNA antibody $<1$, IgM and Ig $G$ anti-cardiolipin antibody $<10$. Arterial Blood Gas analysis on room air showed $\mathrm{pH}$ 7.39, $\mathrm{pCO}_{2} 33, \mathrm{pO}_{2}$ 91, and 97 percent $\mathrm{O}_{2}$ saturation. Initial Troponin I was $0.25 \mathrm{ng} / \mathrm{ml}$ (normal 0-0.5 ng/ml) (Figure 1) and EKG showed ST segment depression in the infero-lateral leads (Figure 1b(i)). Such EKG changes are non-specific as these changes could be seen in the setting of NSTEMI or pulmonary embolism. A chest X-ray was normal.

Her calculated Well's Score for pulmonary embolism was 4.5 points,

\section{Troponin I}

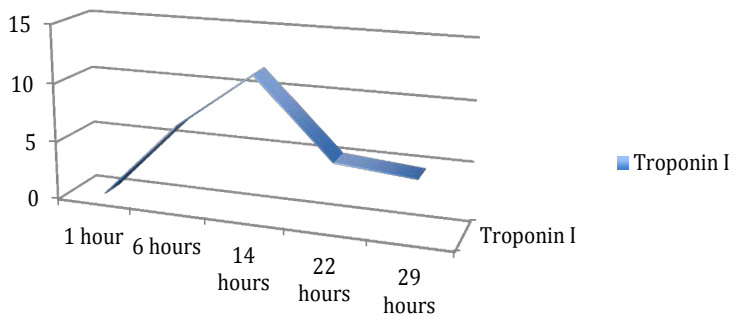

Figure 1a: Trends of Troponin I.

*Corresponding author: Zin W Myint, Medstar Good Samaritan Hospital Baltimore, USA, Tel: 4102368095; E-mail: zinnfang@gmail.com

Received April 07, 2015; Accepted May 27, 2015; Published May 29, 2015

Citation: Myint ZW, Thein KZ, Moe K, Dogar MH, Chow RD (2015) A Case of Post-partum Spontaneous Coronary Artery Dissection with Pulmonary Embolism Survival with Thrombectomy and Stent Implant. J Clin Case Rep 5: 539. doi:10.4172/2165-7920.1000539

Copyright: @ 2015 Myint ZW, et al. This is an open-access article distributed under the terms of the Creative Commons Attribution License, which permits unrestricted use, distribution, and reproduction in any medium, provided the original author and source are credited. 
which placed the patient at moderate risk for PE. As D-dimer testing is not sensitive in this setting, a Computed Tomography Angiogram (CTA) of the chest was then pursued. This revealed a pulmonary embolus involving the right lower lobe subsegmental pulmonary artery, prompting stat administration of $80 \mathrm{mg}$ of enoxaparin.

Because of the abnormal EKG, cardiac enzymes were drawn at 3 hours intervals to rule out a Non-ST Elevation Myocardial Infarction (NSTEMI). Her troponin I increased to $6.83 \mathrm{ng} / \mathrm{ml}$ (normal 0-0.5 ng/ $\mathrm{ml}$ ) over the ensuing six hours and a repeat EKG showed more depth in the $\mathrm{T}$ wave inversion in lateral leads (Figure $1 \mathrm{~b}(\mathrm{ii})$ ). The patient was then treated for acute coronary syndrome with aspirin $325 \mathrm{mg}$, clopidogrel $300 \mathrm{mg}$ loading dose followed by $75 \mathrm{mg}$ daily, atorvastatin $80 \mathrm{mg}$, and metoprolol. Intravenous heparin infusion was started for suspected NSTEMI, and further dosages of enoxaparin were withheld. The patient was upgraded from a telemetry unit to the coronary care unit (CCU) for closer monitoring.

Her troponin I was eventually peaked at $11.71 \mathrm{ng} / \mathrm{ml}$ (normal $0-0.5 \mathrm{ng} / \mathrm{ml}$ ) at 14 hours. An immediate echocardiogram (ECHO) demonstrated moderately decreased left ventricular ejection fraction EF of $30-35 \%$ with antero and antero-septal akinesia. There was no evidence of aortic root dilatation to suggest Marfan's Syndrome.

Emergent cardiac catheterization and angiography revealed a radiolucent linear defect, suggestive of an intimal dissection involving the mid left anterior descending artery (LAD). There was a 99\% stenosis of the true lumen of the mid-LAD, caused by external compression exerted by the hematoma in the false lumen (Figure $2 a$ and $2 b$ ). The estimated LVEF by contrast ventriculography was $30 \%$. Coronary thrombectomy was then performed, followed by placement of a drugeluting stent.

The patient recovered without any further complications and was discharged on a regimen of aspirin, clopidogrel, atorvastatin,

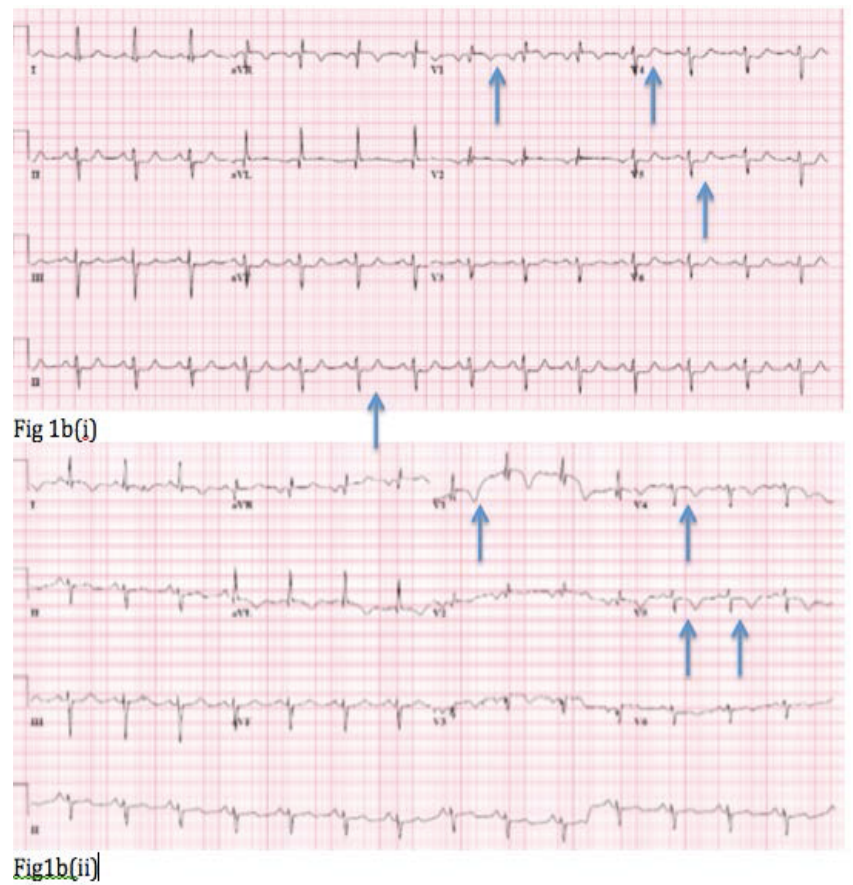

Figure 1b(i): Initial EKG showed ST depression in Lead II, V4, V5. Figure $1 \mathrm{~b}$ (ii): Repeat EKG in 6 hours showed more depth in $T$ wave inversion in $\mathrm{V} 1$, V4, V5 leads.

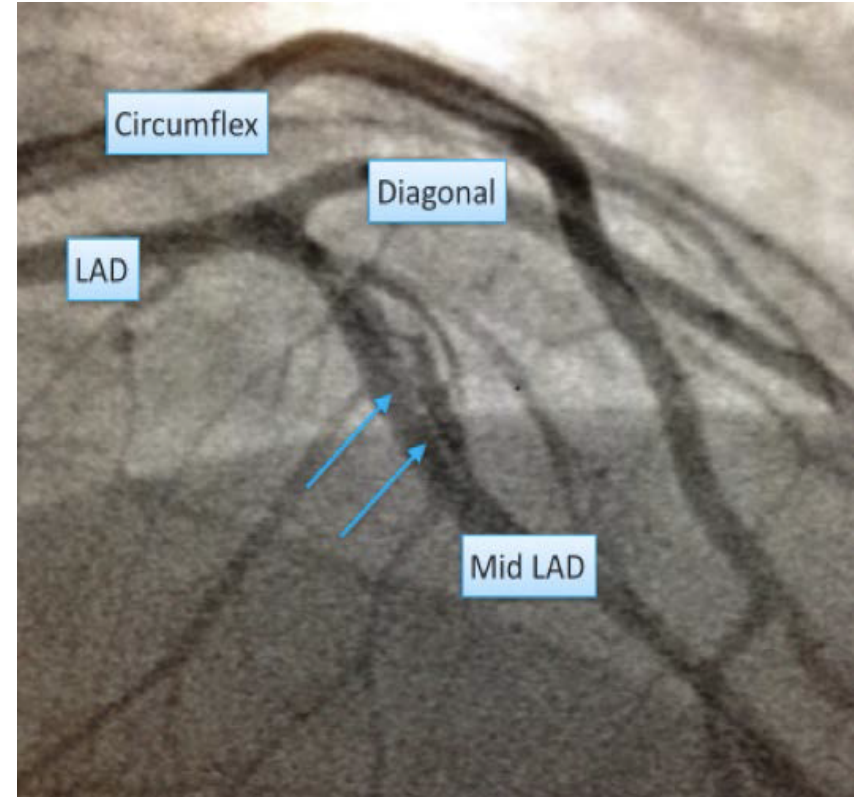

Figure 2a: Diagnostic coronary angiography showed a dissection of the left anterior descending artery (Arrow).

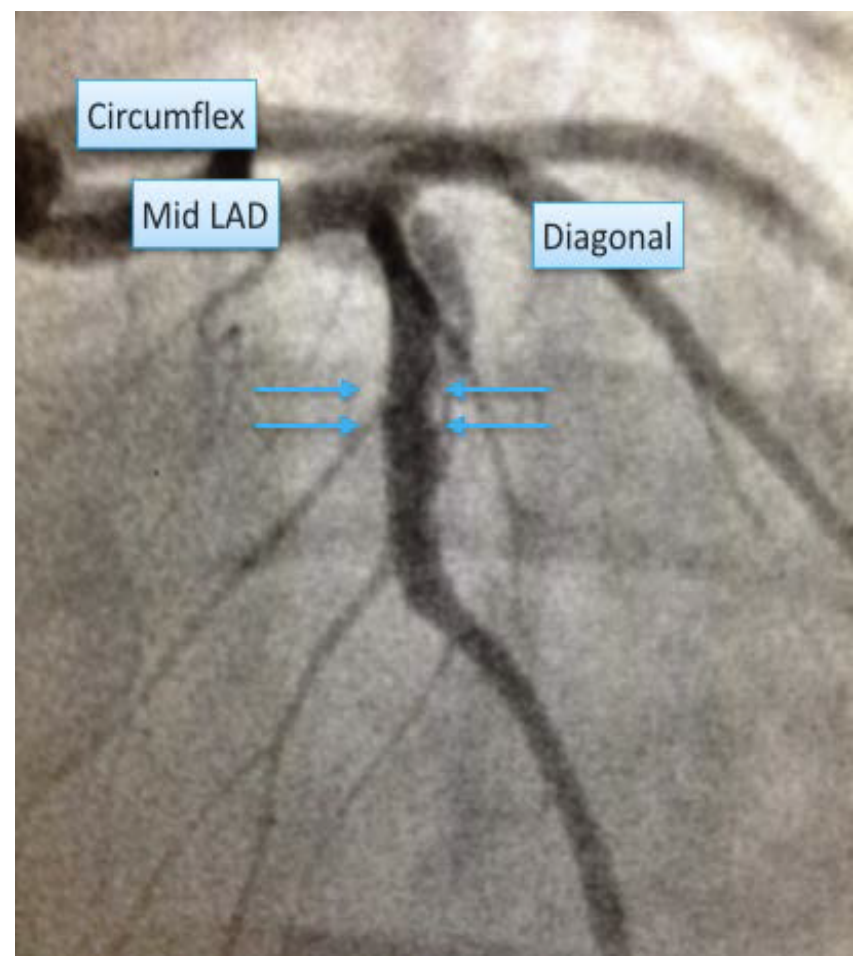

Figure $\mathbf{2 b}$ : There was a $99 \%$ stenosis of the true lumen of the mid-left anterior descending artery caused by external compression by the hematoma in the false lumen.

metoprolol, lisinopril, and warfarin. Her post-hospitalization course was uncomplicated, and the warfarin was terminated after three months. At the time of her six- month follow-up appointment, she was experiencing no chest pain or shortness of breath. A repeat echocardiogram at that time demonstrated that the EF estimated at $60-$ $65 \%$, had returned to normal. 


\section{Discussion}

SCAD, with a reported mortality of up to $80 \%$, is an uncommon cause of acute coronary syndrome [1-5]. It commonly occurs in younger patients, typically pregnant women during their pre-partum or postpartum periods. The increased incidence of SCAD in the pregnancy and the postpartum period is thought to be related to decreased collagen production during pregnancy, which results in weakening of the intima and media of the vessels walls [6]. Increased collagenase activity, hemodynamic factors and eosinophilic periadventitial infiltration involving vasa vasorum have all been proposed to contribute to the vulnerability of vessel wall in such patients [7].

Though SCAD can also occur in the geriatric population, the mechanism here is likely different from that which occurs in younger patients. Geriatric patients typically experience atherosclerotic plaque rupture, with disruption of the intima-media junction [8]. Formation of an intramural hematoma can also result in luminal occlusion and thus myocardial infarction [1]. SCAD has also been described in patients without coronary artery risk factors [9]. These patients may have had secondary causes such as coronary interventions, cardiac surgery, sarcoidosis or isolated fibromuscular dysplasia of the coronary arteries [10]. Finally, a small minority of SCAD patients do not have an identified cause or predisposition; they are termed "spontaneous SCAD" [10].

The most predominant coronary vessel involved in SCAD is the left anterior descending artery $(72 \%)$, as was the case with our patient. However, other vessels can also be involved: left main artery (4\%-8\%), right coronary artery (20\%), and left circumflex coronary artery (4\%) [11]. The diagnosis is usually confirmed by coronary angiography [12]. The common findings are the presence of an intimal flap, two separate communicating lumens, multiple dissecting lines, and an aneurysm communicating with the lumen.

The hypercoagulability associated with pregnancy is related to increased production of pro-coagulants factors like VII, VIII, IX, X, XII, and fibrinogen, and decreased levels of fibrinolytic factors like protein $\mathrm{S}$. Plasminogen activator inhibitor activity is also increased, contributing to thrombophilia [13]. Pelvic and lower extremity venous stasis in this pro-thrombotic milieu will predispose pregnant and post-partum patients to pulmonary embolism. One study showed that the incidence of venous thromboembolism in postpartum women is five times higher than in antepartum cohorts [14]. A case-controlled study demonstrated that sickle cell trait is a risk factor for venous thromboembolism and pulmonary embolism; in that study, sickle cell trait patients carried three times the risk of developing pulmonary embolism and twice the risk of venous thromboembolism than controls [15]. It is possible that sickle cell trait was a contributing factor in the development of PE in our patient.

The mechanism linking SCAD with pulmonary embolism remains poorly understood. In patients with SCAD, pathological examination usually reveals the development of a dissection plane between the media and the adventitia of the affected coronary artery. It has been hypothesized that the inciting event is cystic media necrosis or hemorrhagic disruption of the vasa vasorum [16]. The expansion of the dissected lumen through further bleeding can lead to compression of the true lumen with subsequent myocardial ischemia or infarction [17]. An underlying inflammatory process has also been suggested as a possible mechanism resulting in separation of the arterial wall layers [10]. For example, it has been reported that anti-phospholipid antibody syndrome induces endothelial damage, which can result in spontaneous dissection in the affected arteries [18]. Finally, several authors have proposed that hemodynamic stress can cause increased shear stress on coronary artery walls, thereby causing them to dissect. Indeed, this repeated local stretching of coronary arteries has been suggested as the mechanism by which pulmonary embolism results in an intimal tear [9].

It is possible that underlying occult pulmonary emboli have occurred in pregnant or post-partum women who are diagnosed with SCAD, but remain largely undetected due to lack of clinical suspicion. The clinical focus in such patients would be on acutely managing the SCAD and its sequelae. In summary, we postulate that the pulmonary embolism in our patient resulted from hypercoagulability associated with her post-partum state in combination with her sickle cell trait. Other hypercoagulable states such as lupus, the use of oral contraceptives were ruled out. Due to her persistent troponin elevation, NSTEMI was diagnosed. Cardiac catheterization was subsequently performed and found to have SCAD. The pulmonary embolism then caused increased shear stress on the heart and its coronary vessels, resulting in NSTEMI, and subsequently SCAD.

There are three other reported cases of SCAD associated with PE reported in the English Literature (Table 1). These cases are similar to our case, and the same proposed mechanism may hold in these cases as well; one was a post-partum patient similar to ours.

The approach to treatment of SCAD is still debatable. Available treatment options, all aimed at restoration of perfusion to the myocardium, include the following:

1) Conservative medical therapy with heparin, beta-blockers, nitrates, and anti-platelet agent

2) Balloon angioplasty with either drug eluting or bare metal stents. Typically, interventional stent therapy is reserved for a single vessel disease with a well-localized lesion, with the exception of left main stem disease. Thrombectomy followed by stent placement has not been heretofore described as a treatment option for SCAD. In our case, a suspected thrombus at the proximal dissected artery was seen during cardiac catheterization procedure. Thrombectomy was attempted after placement of a PCI guidewire through the true lumen. A stent was then placed to wall off the true lumen.

3) Surgical therapy with coronary artery bypass graft (CABG) in cases of multi-vessels or involvement of the left main stem artery.

4) Thrombolysis is controversial, as it carries a high risk of bleeding, and is generally avoided [19].

There is no evidence that one approach is superior to another [20].

\begin{tabular}{|c|c|c|c|c|c|}
\hline Author & Presenting symptom & Culprit vessels & Acute coronary syndrome & Vascular complication & Treatment \\
\hline Singh et al. & Chest pain & Proximal left anterior descending artery & STEMI & Bilateral pulmonary embolism & Medical \\
\hline Ozdogru et al. & Chest pain and dyspnea & Right coronary artery & Not associated & Three emboli in pulmonary vasculature & Medical \\
\hline Afonso et al. & Syncope & Distal right coronary artery & NSTEMI & Bilateral pulmonary embolism & Medical \\
\hline
\end{tabular}

Table 1: Previous case reports of combined SCAD and pulmonary embolism. 
The medical, interventional, and surgical management approaches have all been reported to be effective [21,22]. The overall prognosis mandates close follow-up of patients with SCAD, as one cohort study reported a recurrence rate of SCAD as high as $29.4 \%$ in 10 years, predominantly in females [23]. Interestingly, the phenomenon of recurrent SCAD in subsequent pregnancies has not been reported.

\section{Conclusion}

Patients in the antepartum or post-partum period are hypercoagulable and at risk for venous thrombotic events. Clinicians should be aware of the presentation of SCAD and its potential association with pulmonary embolism. SCAD should be included in the differential diagnosis of young patients presenting with chest pain, especially in the presence of hypercoagulable state. In pregnant or post-partum patients with SCAD, the presence of an underlying PE should be considered. Treatment options for SCAD include medical management, balloon angioplasty with stent placement, or surgery. Balloon angioplasty with thrombectomy was a successful approach in our patient. In patients with SCAD, close follow up is recommended due to an overall guarded prognosis.

\section{References}

1. DeMaio SJ Jr, Kinsella SH, Silverman ME (1989) Clinical course and longterm prognosis of spontaneous coronary artery dissection. Am J Cardiol 64: 471-474.

2. Hering D, Piper C, Hohmann C, Schultheiss HP, Horstkotte D (1998) [Prospective study of the incidence, pathogenesis and therapy of spontaneous, by coronary angiography diagnosed coronary artery dissection]. Z Kardiol 87: 961-970.

3. Mohamed HA, Eshawesh A, Habib N (2002) Spontaneous coronary artery dissection--a case report and review of the literature. Angiology 53: 205-211.

4. Jorgensen MB, Aharonian V, Mansukhani P, Mahrer PR (1994) Spontaneous coronary dissection: a cluster of cases with this rare finding. Am Heart J 127: 1382-1387.

5. Koul AK, Hollander G, Moskovits N, Frankel R, Herrera L, et al. (2001) Coronary artery dissection during pregnancy and the postpartum period: two case reports and review of literature. Catheter Cardiovasc Interv 52: 88-94.

6. Appleby CE, Barolet A, Ing D, Ross J, Schwartz L, et al. (2009) Contemporary management of pregnancy-related coronary artery dissection: A single-centre experience and literature review. Exp Clin Cardiol 14: e8-8e16.

7. Austin H, Key NS, Benson JM, Lally C, Dowling NF, et al. (2007) Sickle cell trait and the risk of venous thromboembolism among blacks. Blood 110: 908-912.

8. Celik SK, Sagcan A, Altintig A, Yuksel M, Akin M, et al. (2001) Primary spontaneous coronary artery dissections in atherosclerotic patients. Report of nine cases with review of the pertinent literature. Eur J Cardiothorac Surg 20: 573-576.

9. Siddiqui F, Briasoulis A, Siddiqui S, Rashed A, Afonso L (2014) Spontaneous Distal Right Coronary Artery Dissection in a Patient With Massive Pulmonary Embolism: Thrombolyze or Not? Am J Ther .

10. Gul I, Basar E, Cetinkaya Y, Kasapkara A, Kalay N, et al. (2007) Spontaneous coronary artery dissection and pulmonary thromboembolism: a case report. Int J Cardiol 118: e21-23.

11. Kamineni R, Sadhu A, Alpert JS (2007) Spontaneous coronary artery dissection and pulmonary thromboembolism. a case report. Int J Cardiol 118: e21-e23.

12. Biswas M, Sethi A, Voyce SJ (2012) Spontaneous coronary artery dissection: case report and review of literature. Heart Views 13: 149-154.

13. Appleby CE, Barolet A, Ing D, Ross J, Schwartz L, et al. (2009) Contemporary management of pregnancy-related coronary artery dissection: A single-centre experience and literature review. Exp Clin Cardiol 14: e8-8e16.

14. Heit JA, Kobbervig CE, James AH, Petterson TM, Bailey KR, et al. (2005) Trends in the incidence of venous thromboembolism during pregnancy or postpartum: a 30-year population-based study. Ann Intern Med 143: 697-706.

15. Borczuk AC, van Hoeven KH, Factor SM (1997) Review and hypothesis: the eosinophil and peripartum heart disease (myocarditis and coronary artery dissection)--coincidence or pathogenetic significance? Cardiovasc Res 33: 527-532.

16. Efstratiou A, Singh B (1994) Combined spontaneous postpartum coronary artery dissection and pulmonary embolism with survival. Catherization and cardiovascular diagnosis 31: 29-33.

17. Acikel S, Yildirir A, Muderrisoglu H (2009) Spontaneous coronary artery dissection during as an ischemic stroke in a middle-aged man with anticardiolipin antibodies. Int $\mathrm{J}$ cardiol 134: e53-e55.

18. Kluger BM, Hughes RL, Anderson CA, Hassell KL (2008) Non-traumatic carotid dissection and stroke associated with anti-phospholipid antibody syndrome: report of a case and review of the literature. Neurol India 56: 77-78.

19. Kansara P, Graham S (2011) Spontaneous coronary artery dissection: case series with extended follow up. J Invasive Cardiol 23: 76-80.

20. Biswas M, Sethi A, Voyce SJ (2012) Spontaneous coronary artery dissection case report and review of literature. Heart Views 13: 149-154.

21. McCann AB, Whitbourn RJ (2009) Spontaneous coronary artery dissection: a review of the etiology and available treatment options. Heart Vessels 24: 463465.

22. Shankarappa RK, Panneerselvam A, Dwarakaprasad R, Karur S, Krishnanaik $\mathrm{GB}$, et al. (2009) Spontaneous asymptomatic coronary artery dissection in a young man. J Cardiol 54: 499-502.

23. Tweet MS, Hayes SN, Pitta SR, Simari RD, Lerman A, et al. (2012) Clinica features, management, and prognosis of spontaneous coronary artery dissection. Circulation 126: 579-588. 